\title{
ESPAÇOS, PRÁTICAS: O QUE HÁ DE NOVO NA PSICOLOGIA NO RIO GRANDE DO NORTE? ${ }^{1}$
}

\author{
Oswaldo H. Yamamoto \\ Rosa Angélica Câmara" \\ Fabiana Lima Silva ${ }^{\#}$ \\ Candida Maria B. Dantas ${ }^{\mathbb{I}}$
}

\begin{abstract}
RESUMO. Nos últimos anos, os estudos acerca da realidade da profissão de psicólogo no Brasil têm registrado o que se convencionou denominar de tendências emergentes nos campos de trabalho, com novos locais e práticas inovadoras. O objetivo deste estudo foi, a partir do levantamento dos locais nos quais os psicólogos norte-riograndenses estão inseridos e das atividades neles desenvolvidas, analisar o quadro do estado com relação às mudanças em curso na Psicologia. Um questionário semi-estruturado foi aplicado a 310 dos 631 psicólogos regularmente inscritos no CRP13/Seção RN. Os principais resultados mostram que: (a) embora haja registros de locais antes não ocupados pelos psicólogos do estado, há uma concentração dos profissionais nos locais tradicionais; (b) as atividades desenvolvidas tanto nos "novos" locais quanto nos "convencionais" são aquelas consagradas pela prática tradicional. São levantadas algumas hipóteses explicativas para esse quadro e a situação do estado no que diz respeito às chamadas inovações na Psicologia.
\end{abstract}

Palavras-chave: formação e atuação do psicólogo, Psicologia no Rio Grande do Norte, Psicologia no Brasil

\section{POSITIONS, PRACTICES: WHAT IS NEW IN THE RIO GRANDE DO NORTE'S PSYCHOLOGY?}

\begin{abstract}
Studies about the situation of Brazilian Psychology in the last years have pointed out the presence of psychologists in new positions and the emergence of new practices. The purpose of this paper is to describe the psychologist's working positions and activities performed by them in these places, in order to analyse the situation of Psychology in the state of Rio Grande do Norte (Northeast of Brazil) concerning these innovations. A semistructured questionnaire was applied to 310 out of the 631 psychologists registered in the state. Results show that (a) despite the existence of new positions there is a concentration of professionals in "traditional" places, and (b) the activities performed both in the new and in the "traditional" positions can be classified as "traditional". Some explanations to these conclusions are suggested, and the situation of the state concerning the so-called innovations is discussed.
\end{abstract}

Key words: psychologist's professional training and practice, Psychology in Rio Grande do Norte, Psychology in Brazil.

1 Parte dos dados deste trabalho foi apresentada no I Congresso Norte-Nordeste de Psicologia, em Salvador (BA), em maio de 1999. A pesquisa de origem tem apoio do CNPq, processo 520218/96-5. Agradecemos à psicóloga Èdzia Paula de Medeiros pelo auxílio na coleta e tratamento dos dados.

* Doutor em Educação pela Universidade de São Paulo. Professor Adjunto do Departamento de Psicologia da Universidade Federal do Rio Grande do Norte. E-mail: ohy@uol.com.br.

Endereço para correspondência: Av. Estrela do Mar, 2267 - Ponta Negra - 59090-400 -Natal - RN - Telefone: (084)236.2477 Fax: (084)236.3485 E-mail: ohy@uol.com.br

\# Psicóloga e aluna do Curso de Especialização em Psicologia, da Universidade Federal do Rio Grande do Norte.

Il Bolsista de Iniciação Científica do CNPq, estudante do Curso de Psicologia da Universidade Federal do Rio Grande do Norte. 


\section{INTRODUÇÃOO}

A legislação que regula a profissão de psicólogo (Lei 4.119/62 e Decreto 53.464/64) estabelece um rol de funções, umas mais definidas (realizar diagnóstico psicológico, orientação e seleção profissional, orientação psicopedagógica entre outros), outras menos (como exemplo a utilização de métodos e técnicas psicológicos com o objetivo de solucionar problemas de ajustamento).

Conquanto não estabelecendo áreas de atuação, a Lei 4.119/62 reconhece especialidades no campo aplicado da Psicologia: no seu artigo 19 estabelece o direito ao registro aos portadores de certificados e diplomas de especialista em Psicologia Educacional, Psicologia Clínica e Psicologia Aplicada ao Trabalho.

De fato, o que os textos legais fazem é se sobreporm a uma configuração já estruturada nessas especialidades. Em 1953, em documento endereçado ao Ministro da Educação, a Associação Brasileira de Psicotécnica afirmava ter realizado um levantamento constatando a existência de centenas de pessoas em atividade profissional em três ramos capitais: "Psicotécnica Escolar, Psicotécnica do Trabalho e Psicologia Clínica" (Mello, 1975, p.131).

Mello (1975), embora reconhecendo a excessiva simplificação imposta pela classificação nas áreas mencionadas pela lei, toma-a por base no estudo sobre a conformação profissional dos psicólogos paulistas, traçando um perfil da profissão, que mostra uma notável concentração de psicólogos exercendo atividades na área Clínica (125 dos 158 psicólogos), secundados pelas áreas Industrial (31) e Escolar (27) excluídos aí aqueles dedicados ao ensino da Psicologia.

Tomando como base o único levantamento exaustivo cobrindo todo o território nacional, empreendido pelo Conselho Federal de Psicologia e empregando basicamente a mesma nomenclatura, Bastos (1988) confirma a tendência estabelecida pelo estudo de Mello: 55,3\% dos psicólogos têm seu emprego principal na área Clínica, contra 19,2\% e $11,7 \%$ das áreas Organizacional e Escolar, respectivamente.

No âmbito norte-riograndense, estudos também confirmam o mesmo quadro, com predominância bastante acentuada pelo campo Clínico (68\%), contra $14 \%$ e $10 \%$ das áreas do Trabalho e Escolar (Yamamoto, Siqueira \& Oliveira, 1997).

Se, por um lado, parece inquestionável que a configuração profissional da Psicologia no Brasil nos anos que se seguem a sua regulamentação obedece ao padrão verificado nos estudos de Mello (1975), Bastos
(1988) e Yamamoto e cols., (1997), por outro fica evidente, conforme assinalado pelos próprios autores, a imprecisão dessa taxionomia.

Tal imprecisão é potencializada, nos últimos anos, pela perspectiva de que, passado o primeiro momento de consolidação da profissão, o campo profissional vai sendo reformatado pela presença, cada vez mais freqüente, de trabalhos que não podem ser tranqüilamente classificados nas categorias tradicionais: são as chamadas "práticas emergentes" e os "novos espaços de atuação" (Conselho Federal de Psicologia [CFP], 1992; 1994).

Evidentemente, tais mudanças - comportanto tanto a expansão pura e simples do campo profissional pela ocupação de novos espaços profissionais quanto as inovações introduzidas no seio mesmo daqueles campos tradicionais do psicólogo - demandam um monitoramento próximo.

Em que pese o fato de que inovações por si mesmas não avalizam a qualidade do trabalho, nem tampouco garantem relevância social (Yamamoto \& Campos, 1997), o volume das críticas e a insatisfação com a profissão (Bastos, 1988; Bock, 1999; Botomé, 1979; Campos, 1983; Figueiredo, 1989; Mello, 1975; Patto, 1984; Yamamoto, 1987, entre outros) impõem um olhar atento para tais movimentações no campo profissional da Psicologia, pela sua eventual potencialidade de abertura de perspectivas (mais) estimulantes para a profissão.

No mapeamento da profissão realizado no Rio Grande do Norte, já referido (Yamamoto e cols., 1997), além dos campos tradicionais era possível vislumbrar, ainda que de maneira débil, um certo nível de expansão do campo profissional.

Esta era traduzida pela inserção do psicólogo em locais que, conquanto nacionalmente não se constituíssem propriamente em novidades - como ilustração, destacaríamos a presença do psicólogo em Delegacias de Defesa da Mulher, em Varas da Infância e da Juventude, no Serviço de Vigilância Sanitária e no Centro Integrado de Atenção ao Idoso -, no âmbito local isto configurava uma mudança digna de registro.

As questões a serem colocadas com relação a esses dados são da mesma natureza da assinalada anteriormente: sem entrar no mérito da constituição ou não de uma nova área, o que representa essa ampliação do espaço profissional?

Partindo de uma lógica diversa da classificação do campo profissional em áreas, o objetivo deste estudo foi verificar as atividades desenvolvidas pelos psicólogos norte-riograndenses nos diferentes locais de inserção. Ao estabelecer tal relação, buscou-se um 
olhar mais detido no que existe de novidade no desenvolvimento de ações nos chamados novos contextos de atuação do psicólogo.

\section{MATERIAIS E MÉTODOS}

Este estudo foi conduzido com 310 psicólogos (87,7\% do sexo feminino; $51 \%$ na faixa de $26-35$ anos de idade; $78 \%$ formados na Universidade Federal do Rio Grande do Norte; $88 \%$ desenvolvendo, então, trabalhos em Psicologia), representando 49,2\% dos 631 psicólogos regulamente inscritos na Seção $\mathrm{RN}$ do Conselho Regional de Psicologia, $13^{\mathrm{a}}$. Região, no período da coleta.

Um questionário semi-estruturado, contendo três partes, foi aplicado por ocasião da eleição para a recomposição do CRP-13, na sede de Natal (RN). Os dados a serem apresentados aqui correspondem aos obtidos nas duas partes iniciais, a saber, caracterização sócio-demográfica e atividades desenvolvidas nos locais de trabalho. O rol de atividades apresentado teve por base a listagem contida no estudo de Carvalho (1988), com pequenas alterações e opção para acréscimos por parte dos respondentes. Os dados de uma terceira parte, com levantamento das expectativas com relação à atuação do CRP-13, não estão sendo considerados aqui.

\section{RESULTADOS E DISCUSSÃO}

\section{Novos espaços?}

Um dos aspectos centrais nos estudos sobre as mudanças em curso na Psicologia no Brasil é a ampliação dos espaços profissionais. A Tabela 1 apresenta dados referentes aos locais nos quais os psicólogos norteriograndenses estão atuando (mais de dez referências), para termos uma primeira aproximação das eventuais inovações na prática profissional.

Tabela 1. Locais de trabalho dos psicólogos norteriograndenses.

\begin{tabular}{lcc}
\hline Locais de atuação & $\mathbf{n}^{*}$ & $\mathbf{\%}$ \\
\hline Clínicas Psicológicas & 149 & 40,2 \\
Unidades Básicas de Saúde & 55 & 14,8 \\
Empresas/Organizações & 35 & 9,4 \\
Instituições de Ensino Superior (IES)** & 30 & 8,1 \\
Hospitais & 25 & 6,7 \\
Instituições Educacionais & 21 & 5,7 \\
Outros & 56 & 15,1 \\
\hline Total & 371 & 100,0 \\
\hline
\end{tabular}

* Múltiplas respostas admitidas

** Incluindo serviços de psicologia/clínicas-escola
Se agregarmos novamente esses dados considerando, grosso modo, as áreas de atuação conforme a classificação do Conselho Regional de Psicologia-6a . Região (CRP-6, 1995), apenas para possibilitar uma comparação com a literatura, verificaremos que aproximadamente $60 \%$ dos psicólogos norte-riograndenses situar-se-iam no campo da Saúde, $14 \%$ no setor Educacional e 9\% em Trabalho. Em termos gerais, a distribuição obtida é semelhante à do CRP-6 (1995) - 54,67\%; 17,86\% e $13,05 \%$ respectivamente, e dos demais estudos, de âmbito nacional e local (CFP, 1988; Yamamoto e cols., 1997).

Uma segunda observação a fazer com relação a esses dados diz respeito à existência de cerca de $15 \%$ das respostas que não puderam ser enquadradas nas categorias mais amplas. Estes locais, com respectivas freqüências de menções são: "Escritórios de consultorias" (autônomo), 7; "Centro Estadual de Educação Especial" e "NAPS/CAPS (Núcleos de Assistência Psicossocial/Centros de Assistência Psicossocial", 5; "Centro de Reabilitação Profissional (INSS)" e "CRI" (Centro de Reabilitação Infantil). 4; "SESI (Serviço Social da Indústria)" e "IPE (Instituto de Previdência dos Servidores do Estado)", 3; "COOPSI (Cooperativa dos Psicólogos)" e "FUNDAC" (Fundação Estadual da Criança e do Adolescente/RN) e "FUNPEC" (Fundação de Pesquisa da UFRN), 2; e 19 outros com apenas uma indicação (incluindo locais tão diversos como a "Penitenciária de Alcaçus" e o "Teatro Sandoval Wanderley").

Esses dados, de início, nos mostram que, embora não alterem de forma significativa a distribuição provisória de áreas que apresentamos anteriormente, o trabalho realizado em alguns desses "novos" locais pode ser enquadrado dentro daquela classificação tripartite citada anteriormente. Para exemplificar, o local mais freqüente indicado é o que denominamos "Escritórios de consultoria", que distinguimos de "Empresas/organizações", nas quais o psicólogo tem vínculo empregatício. Da mesma forma, os trabalhos realizados em locais como os NAPS/CAPS seriam reenquadrados como pertencendo ao campo da Saúde.

Outra observação que esses dados sugerem é que vários desses locais, embora pouco freqüientes ou novos no estado, não têm esse mesmo caráter em outros centros em que os serviços psicológicos se encontram mais difundidos e inseridos. São os casos de trabalhos em penitenciárias, em juizados e, principalmente, em diversas instituições de reabilitação. 
Esses conjuntos de dados confirmam nosso entendimento, expresso anteriormente, a respeito da dificuldade de enquadramento de trabalhos em "áreas". Por outro lado, embora esses dados confirmem que o cenário da Psicologia potiguar está se alterando com relação aos levantamentos anteriores (Yamamoto, 1988; Yamamoto e cols., 1997), torna-se muito difícil definir o caráter de novidade exclusivamente através dos locais.

\section{Práticas emergentes?}

A questão seguinte a ser respondida diz respeito à forma pela qual estes espaços, sejam "tradicionais", sejam "novos", estão sendo ocupados pelos psicólogos. A Tabela 2 apresenta a relação das atividades desenvolvidas pelos psicólogos norteriograndenses.

Tabela 2. Atividades desenvolvidas pelos psicólogos norte-riograndenses.

\begin{tabular}{|c|c|c|}
\hline Atividades & $\mathbf{n}^{*}$ & $\% * *$ \\
\hline Psicoterapia & 227 & 73,2 \\
\hline Orientação de pais & 164 & 52,9 \\
\hline Diagnóstico psicológico & 123 & 39,7 \\
\hline Aconselhamento psicológico & 103 & 33,2 \\
\hline Aplicação de testes & 94 & 30,3 \\
\hline Atuação junto à comunidade & 71 & 22,9 \\
\hline Treinamento e Desenvolvimento de pessoal & 63 & 20,3 \\
\hline Orientação sexual & 62 & 20,0 \\
\hline Planejamento e execução de projetos & 57 & 18,4 \\
\hline Seleção e orientação de profissional & 53 & 17,1 \\
\hline Atendimento de distúrbios de aprendizagem & 51 & 16,5 \\
\hline Supervisão didática a outros psicólogos & 43 & 13,9 \\
\hline Orientação psicopedagógica & 40 & 12,9 \\
\hline Orientação vocacional & 40 & 12,9 \\
\hline Assistência materno-infantil & 40 & 12,9 \\
\hline Consultoria organizacional & 31 & 10,0 \\
\hline Avaliação curricular & 19 & 6,1 \\
\hline Educação e reeducação motora & 15 & 4,8 \\
\hline Ensino & 4 & 1,3 \\
\hline Dinâmica de grupo & 3 & 1,0 \\
\hline Capacitação de pessoal & 2 & 0,6 \\
\hline Gerência de serviço público & 2 & 0,6 \\
\hline Orientação aos professores & 2 & 0,6 \\
\hline Atendimento em grupo & 2 & 0,6 \\
\hline Supervisão de estágio & 1 & 0,3 \\
\hline Grupoterapia com dependentes químicos & 1 & 0,3 \\
\hline Atuação junto a equipe interdisciplinar & 1 & 0,3 \\
\hline Aconselh. orientação de pessoal em Medicina Social & 1 & 0,3 \\
\hline Preparação cirúrgica & 1 & 0,3 \\
\hline Acompanhamento a crianças & 1 & 0,3 \\
\hline $\begin{array}{l}\text { Reabilitação e psicoterapia com crianças portadoras de } \\
\text { deficiências }\end{array}$ & 1 & 0,3 \\
\hline Reabilitação & 1 & 0,3 \\
\hline
\end{tabular}

* Respostas múltiplas admitidas
** Percentual das atividades com relação ao total de psicólogos (310)

O rol final de atividades, consideradas aquelas contidas no estudo de Carvalho (1988), que serviu de base, e os acréscimos, totalizou 32 diferentes modalidades, com um total de 1.319 ocorrências.

Esta tabela apresenta, na primeira coluna numérica, o total de menções com relação a cada uma das atividades referidas; na segunda, os percentuais calculados com relação ao total de psicólogos, e não de atividades.

O dado de maior impacto é que quase três quartos dos psicólogos norte-riograndenses, independentemente dos locais em que atuam, desenvolvem trabalhos psicoterápicos. Se tomarmos as 11 atividades mais citadas (mais de 50 referências), totalizando mais de $80 \%$ do total de referências, observaremos que, talvez com a exceção de "Atuação junto à comunidade" (que, além de vaga, dificilmente pode ser considerada uma inovação), elas dizem respeito a modalidades tradicionalmente desenvolvidas pelos psicólogos brasileiros.

Optamos por apresentar os dados da forma mais desagregada possível, de forma a permitir uma visualização das eventuais inovações com relação às práticas. Se fôssemos definindo categorias mais amplas de atividades, certamente diversas delas se somariam às mais citadas, diminuindo ainda mais o leque das possíveis práticas novas.

Portanto, se existem práticas emergentes no trabalho dos psicólogos norte-riograndenses, elas certamente não estão sendo desenvolvidas de forma maciça, mas apenas incidentalmente (menos de $10 \%$ de citações). Mas, se olharmos para a parte inferior da Tabela 2 verificaremos que as atividades ali arroladas também não são, talvez salvo algumas exceções, novidades na Psicologia. Desta forma, tomando por base os dados deste estudo, dificilmente poderíamos concluir que existem práticas inovadoras sendo desenvolvidas na Psicologia norte-riograndense.

\section{Práticas emergentes nos novos contextos de atuação?}

Partimos para esta seção com dois conjuntos nada estimulantes de constatações: nem há tantos locais novos, nem tantas práticas emergentes. Todavia, o quadro não ficaria completo se não considerássemos as atividades desenvolvidas pelos psicólogos nos locais de trabalho, novamente tanto os "tradicionais" quanto os "novos". Devido à grande massa de dados, selecionamos, para esta 
análise, apenas alguns dos locais de trabalho referidos pelos profissionais.

Assim, a Tabela 3 apresenta os dados referentes às atividades dos 149 psicólogos que trabalham em "Clínicas psicológicas":

Tabela 3. Atividades desenvolvidas pelos psicólogos que trabalham em Clínicas Psicológicas (n=149).

\begin{tabular}{lcc}
\hline Atividade & n & \% \\
\hline Psicoterapia & 137 & 91,9 \\
Orientação de pais & 79 & 53,0 \\
Diagnóstico psicológico/ avaliação & 67 & 45,0 \\
Aplicação de testes/ exame psicotécnicos & 32 & 21,5 \\
Aconselhamento psicológico & 29 & 19,5 \\
Orientação vocacional & 24 & 16,1 \\
Atendimento de distúbios de aprendizagem & 23 & 15,4 \\
Supervisão didática a outros psicólogos & 20 & 13,4 \\
Seleção e orientação profissional & 15 & 10,1 \\
Orientação Psicopedagógica & 13 & 8,7 \\
Assistência materno-infantil & 13 & 8,7 \\
Orientação sexual & 13 & 8,7 \\
Treinamento e desenvolvimento de pessoal & 8 & 5,4 \\
Consultoria organizacional & 6 & 4,0 \\
Atuação junto à comunidade & 6 & 4,0 \\
Educação e reeducação psicomotora & 5 & 3,4 \\
Planejamento e execução de projetos & 4 & 2,7 \\
Avaliação curricular & 2 & 1,3 \\
Reabilitação e psicoterapia com crianças portadoras de & & \\
deficiências & 1 & 0,7 \\
Dinâmica de grupo & 1 & 0,7 \\
\hline
\end{tabular}

As atividades mais freqüentes não causam surpresa: as diversas modalidades de diagnóstico psicológico e psicoterapia são as mesmas descritas já no estudo de Mello (1975). Contudo, se percorrermos a lista com atenção verificaremos que existem atividades que não seriam esperadas nesses locais, tais como "avaliação curricular", "treinamento e desenvolvimento de pessoal" ou, mesmo, "atuação junto à comunidade".

Estes dados confirmam a interpretação de Mello (1975) sobre a Psicologia Clínica: a confusão entre o que denominam de "área de aplicação da ciência psicológica" com seu local de trabalho (p. 48). Desta forma, a clínica ou o consultório acabam sendo um simples local de trabalho no qual o psicólogo presta serviços como autônomo, independentemente da natureza do trabalho. Nos dados anteriores, atividades que seriam normalmente classificadas como pertencendo à área organizacional/trabalho ou educacional/escolar são exercidas nas "Clínicas Psicológicas" - aliás, a mesma realidade constatada na passagem das décadas de setenta e oitenta por Mello (1975).
A Tabela 4 apresenta os dados referentes às atividades desenvolvidas pelos 35 psicólogos que trabalham em Empresas/organizações: 
Tabela 4. Atividades desenvolvidas pelos psicólogos que trabalham em Empresas/Organizações $(n=35)$.

\begin{tabular}{lrc}
\hline Atividade & n & \% \\
\hline Treinamento e desenvolvimento de pessoal & 24 & 68,6 \\
Aplicação de testes/ exame psicotécnicos & 24 & 68,6 \\
Seleção e orientação profissional & 22 & 62,9 \\
Planejamento e execução de projetos & 12 & 34,3 \\
Aconselhamento psicológico & 11 & 31,4 \\
Avaliação curricular & 8 & 22,9 \\
Diagnóstico psicológico/ avaliação & 7 & 20,0 \\
Consultoria organizacional & 6 & 17,1 \\
Atuação junto à comunidade & 3 & 8,6 \\
Atendimento de distúbios de aprendizagem & 2 & 5,7 \\
Orientação de pais & 2 & 5,7 \\
Orientação Sexual & 2 & 5,7 \\
Pesquisa & 2 & 5,7 \\
Orientação Psicopedagógica & 1 & 2,9 \\
Supervisão didática a outros psicólogos & 1 & 2,9 \\
\hline
\end{tabular}

A despeito de algumas atividades inesperadas, mas explicáveis pela abrangência da categoria "Empresas/Organizações", a relação apresentada na Tabela 4 é bastante mais homogênea que a anterior, tanto no que diz respeito à amplitude (15 nesta, contra 20 em "Clínicas"), quanto pelas atividades desenvolvidas. Observe-se, comparando estes dados com os da Tabela 2 que, conquanto este seja o segundo local de maior concentração de psicólogos, nenhum dos quase $75 \%$ dos que praticam psicoterapia estão aqui.

A Tabela 5 diz respeito às atividades dos psicólogos vinculados a instituições educacionais formais, excetuando-se as de nível superior.

Tabela 5. Atividades desenvolvidas pelos psicólogos que trabalham em Instituições educacionais $(n=21)$.

\begin{tabular}{lcc}
\hline Atividade & n & \% \\
\hline Orientação de pais & 18 & 85,7 \\
Orientação Psicopedagógica & 13 & 61,9 \\
Atendimento de distúbios de aprendizagem & 7 & 33,3 \\
Orientação vocacional & 6 & 28,6 \\
Treinamento e desenvolvimento de pessoal & 6 & 28,6 \\
Planejamento e execução de projetos & 6 & 28,6 \\
Avaliação curricular & 5 & 23,8 \\
Orientação Sexual & 5 & 23,8 \\
Aconselhamento psicológico & 5 & 23,8 \\
Seleção e orientação profissional & 4 & 19,0 \\
Diagnóstico psicológico/ avaliação & 3 & 14,3 \\
Educação e reeducação psicomotora & 2 & 9,5 \\
Reunião com professores & 2 & 9,5 \\
Aplicação de testes/ exame psicotécnicos & 1 & 4,8 \\
Psicoterapia & 1 & 4,8 \\
Assistência materno-infantil & 1 & 4,8 \\
Supervisão didática a outros psicólogos & 1 & 4,8 \\
\hline
\end{tabular}

O conjunto de atividades desenvolvido pelos psicólogos no âmbito escolar é, dos locais até aqui examinados, o mais previsível. A presença de atividades típicas do contexto organizacional, embora não usual, pode ser compreendido se entendida a instituição escolar como uma organização (veja-se, por exemplo, as propostas de Zanelli, 1994, para o trabalho nas organizações). Por outro lado, atividades próprias do campo clínico (como diagnóstico e psicoterapia) não são novidades; antes, são, talvez, resquícios do que se convencionou chamar de "Psicologia escolar clínica" na escola (Andaló, 1984; Maluf, 1994). Um outro ponto a destacar é a ausência, explicável talvez pelos limites impostos pelo instrumento utilizado, de modalidades de ação inovadoras, como as institucionais (Maluf, 1994) ou envolvendo trabalhos em equipes multiprofissionais (Correia \& Campos, 2000), para citar dois exemplos.

Examinemos agora, para finalizar, um local em que a presença do psicólogo, se não é propriamente uma novidade, também não pode ser enquadrada como fazendo parte do perfil tradicional: as Unidades Básicas de Saúde (Tabela 6).

Tabela 6. Atividades desenvolvidas pelos psicólogos que trabalham em Unidades Básicas de Saúde $(n=55)$.

\begin{tabular}{lrr}
\hline Atividade & n & \% \\
\hline Psicoterapia & 39 & 70,9 \\
Orientação de pais & 30 & 54,5 \\
Atuação junto à comunidade & 29 & 52,7 \\
Aconselhamento psicológico & 20 & 36,4 \\
Orientação sexual & 16 & 29,1 \\
Assistência materno-infantil & 14 & 25,5 \\
Diagnóstico psicológico/avaliação & 12 & 21,8 \\
Planejamento e execução de projetos & 10 & 18,2 \\
Atendimento de distúbios de aprendizagem & 9 & 16,4 \\
Treinamento e desenvolvimento de pessoal & 7 & 12,7 \\
Orientação psicopedagógica & 4 & 7,3 \\
Aplicação de testes/exame psicotécnicos & 3 & 5,5 \\
Supervisão didática a outros psicólogos & 3 & 5,5 \\
Educação e reeducação psicomotora & 2 & 3,6 \\
Gerência de serviço público & 2 & 3,6 \\
Dinâmica de grupo & 2 & 3,6 \\
Orientação vocacional & 1 & 1,8 \\
\hline
\end{tabular}

Os dados da Tabela 6 são bastante elucidativos: se bem que a atuação na rede pública de saúde seja de reconhecida importância para o profissional de Psicologia, o que os dados sugerem é que a prática do psicólogo norte-riograndense nesse local, considerando o conjunto, não se distingue das modalidades desenvolvidas em consultórios particulares. Há, é certo, tanto atividades que se afastam dessas práticas tradicionais (como a indefinida "atuação junto à comunidade"), quanto outras 
que seriam mais próprias da saúde pública (como a "assistência materno-infantil", para tomar um exemplo).

\section{Algumas questões sobre as inovações na prática do psicólogo}

Poderíamos prosseguir apresentando os dados dos demais locais, mas isso não somente tornaria esta análise demasiadamente longa, quanto seria desnecessário: os aspectos principais já estão claros, neste ponto.

De fato, tanto no rol de atividades desempenhadas quanto, de forma mais dramática, no confronto com os dados nacionais apresentados por Carvalho (1988), fica evidenciado que, se existem aspectos de novidade na ação dos psicólogos potiguares, elas não se encontram nas atividades desempenhadas mas, eventualmente, nos locais em que trabalham.

Conforme Bastos (1988), analisando os dados do levantamento nacional, a baixa diversificação das atividades dos psicólogos poderia estar relacionada à também restrita "diversidade de situações, de contextos, de questões com que o psicólogo está lidando" (p. 234). Por outro lado, Carvalho (1988) assinalava a importância de ampliar o conceito do atuar psicológico como contrapartida da abertura de espaços por parte da sociedade.

Os dados do presente estudo, no entanto, parecem indicar um quadro um pouco menos otimista no que concerne à introdução de inovações: mesmo no confronto com situações novas, os psicólogos norteriograndenses estão reiterando as práticas consagradas nos locais ditos "tradicionais". Ou seja, o confronto com novas realidades não parece estar expondo aquilo que Campos (1983) denominava de insuficiências dos modelos teóricos tradicionais da Psicologia em dar conta das novas realidades, promovendo o desenvolvimento de práticas inovadoras, mas uma reiteração, eventualmente sem a necessária consideração das circunstâncias e das questões envolvidas, de práticas tradicionais nesses novos contextos de atuação ${ }^{2}$.

2 Na direção de eventuais mudanças desse quadro, é necessário destacar a aprovação da Resolução 014/00 do Conselho Federal de Psicologia, de 22 de dezembro de 2000, instituindo o título profissional de Especialista em Psicologia (nos campos da Psicologia Escolar/Educacional; Organizacional e do Trabalho; do Trânsito; Jurídica; de Esporte; Hospitalar; Clínica; Psicopedagogia e Psicomotricidade). As conseqüências do estabelecimento de tal registro ainda não foram suficientemente estudadas e nem faz parte dos objetivos deste estudo. Mas, é impossível não destacar que, ao mesmo tempo em que pode qualificar a prática profissional pela exigência de uma formação continuada, pode cristalizar os campos de atuação,
As questões que este estudo levanta dizem respeito, fundamentalmente, a dois pontos conectados. Em primeiro lugar, qual a adequação (e sob que perspectiva analítica) das atividades desenvolvidas pelos psicólogos face aos novos contextos de atuação? A questão não comporta uma resposta linear, posto que envolve uma ampla gama de realidades. A hipótese que este estudo nos permite levantar é que, seja quais forem as características das novas demandas, talvez pela limitação do repertório teóricotécnico do psicólogo, a sua atuação é definida e conduzida sem a necessária consideração dos aspectos envolvidos nesses novos contextos. A resposta desta primeira questão, sobretudo, se confirmada a suposição enunciada, coloca a segunda, sobre os requisitos da formação do psicólogo. São questões em aberto, para as quais necessitamos de um olhar mais aguçado, sobretudo partindo do que denominamos de práticas em novos contextos.

Essas reflexões nos remetem a uma questão crucial, que diz respeito ao significado das inovações na Psicologia. Reiteramos que o "novo" em si não configura, necessariamente, nem um avanço do ponto de vista teórico-metodológico, nem uma prática socialmente mais conseqüente (Yamamoto \& Campos, 1997). Além disso, sempre é conveniente lembrar que a atuação profissional, como um processo multifário, requer a consideração das suas diversas facetas e determinações. A caracterização dos novos contextos de atuação idem. Estamos, aqui, considerando apenas dois desses aspectos, os postos de trabalho e as atividades.

Tendo em mente tais limitações de análise, o problema em jogo aqui, como em todas as discussões sobre os modelos de atuação, sobretudo aquelas relativas à superação da prática excludente e elitista que tipificava o "modelo médico" de atuação do psicólogo, diz respeito ao que Carvalho (1988, p. 235) denomina de "potencial de utilidade e de contribuição à sociedade".

contribuindo para uma maior fragmentação teórico-prática da Psicologia. O rebatimento da instituição do registro de especialista sobre a formação (que se pretende generalista) é outra questão em aberto, sobretudo porque se soma à exigência de adaptação dos cursos às Diretrizes Curriculares, decorrente da aprovação da nova Lei de Diretrizes e Bases da Educação Nacional (LDB). É importante assinalar que as Diretrizes Curriculares, propostas pela Comissão de Especialistas instituída pelo Ministério da Educação, e que devem substituir o Currículo Mínimo dos cursos de formação em Psicologia, ainda não foram aprovadas pelo Conselho Nacional de Educação. Para uma análise dessas Diretrizes e sua relação com a LDB, ver Yamamoto (2000). 
Lamentavelmente, nem é tal premissa fundamental para o estabelecimento e o desenvolvimento de uma profissão (Freidson, 1970; 1998), nem tampouco o treinamento para lidar com questões relativas à prática da cidadania é parte da formação profissional do psicólogo (Spink, 1992).

\section{REFERÊNCIAS BIBLIOGRÁFICAS}

Andaló, C. S. de A. (1984). O papel do psicólogo escolar. Psicologia: Ciência e Profissão, 1, 43-46.

Bastos, A. V. B. (1988). Áreas de atuação: em questão o nosso modelo profissional. Em CFP, Quem é o psicólogo brasileiro? (pp. 163-193). São Paulo: Edicon.

Bock, A. M. B. (1999). As aventuras do Barão de Münchhausen na psicologia. São Paulo: EDUC.

Botomé, S. P. (1979). A quem nós, psicólogos, servimos de fato? Psicologia, $\underline{5}, 1-15$.

Campos, R. H. (1983). A função social do psicólogo. Educação \& Sociedade, 16, 74-84.

Carvalho, A. M. A. (1988). Atuação psicológica: uma análise das atividades desempenhadas pelos psicólogos. Em CFP, Quem é o psicólogo brasileiro? (pp. 217-235). São Paulo: Edicon.

Conselho Federal de Psicologia. (1988). Quem é o psicólogo brasileiro? São Paulo: Edicon.

Conselho Federal de Psicologia. (1992). Psicólogo brasileiro: Construção de novos espaços. Campinas: Átomo.

Conselho Federal de Psicologia. (1994). Psicólogo brasileiro: Práticas emergentes e desafios para a profissão. São Paulo: Casa do Psicólogo.

Conselho Regional de Psicologia, 6 ${ }^{\mathrm{a}}$. Região. (1995). Psicologia: Formação, atuação profissional e mercado de trabalho (Estatísticas 1995). São Paulo: Autor.

Correia, M., \& Campos, H. R. (2000). Psicologia Escolar: histórias, tendências e possibilidades. Em O. H. Yamamoto \& A. Cabral Neto (Orgs.), O psicólogo e a escola (pp.5997). Natal: EDUFRN.

Figueiredo, M. A. C. (1989). O trabalho alienado \& o psicólogo do trabalho: algumas questões sobre o papel do psicólogo no controle da produção capitalista. São Paulo: Edicon.
Freidson, E. (1970). Profession of Medicine: A study of the Sociology of applied knowledge. Chicago: The University of Chicago Press.

Freidson, E. (1998). Renascimento do profissionalismo. São Paulo: EDUSP.

Mello, S. L. (1975). Psicologia e profissão em São Paulo. São Paulo: Ática.

Maluf, M. R. (1994). Formação e atuação do psicólogo na educação: dinâmica de transformação. Em CFP, Psicólogo brasileiro: práticas emergentes e desafios para a formação (pp. 157-200). São Paulo: Casa do Psicólogo.

Patto, M. H. S. (1984). Psicologia e ideologia: uma introdução crítica à Psicologia Escolar. São Paulo: T. A. Queiroz.

Spink, P. K. (1992). Saúde mental e trabalho: o bloqueio de uma prática acessível. Em F. C. B. Campos (Org.), Psicologia e saúde: repensando práticas (pp. 91-102). São Paulo: Hucitec.

Yamamoto, O. H. (1987). A crise e as alternativas da Psicologia. São Paulo: Edicon.

Yamamoto, O. H. (1988). Apontamentos para um estudo da psicologia em Natal: áreas de atuação e seus determinantes. Revista de Psicologia, 1, 3-13.

Yamamoto, O. H. (2000). A LDB e a Psicologia. Psicologia: Ciência e Profissão, 20 (4), 30-37.

Yamamoto, O. H., \& Campos, H. R. (1997). Novos espaços, práticas emergentes: um novo horizonte para a psicologia brasileira? Psicologia em Estudo, 2 (2), 89-111.

Yamamoto, O. H., Siqueira, G. S., \& Oliveira, S. C. C. (1997). A Psicologia no Rio Grande do Norte: Caracterização geral da formação acadêmica e do exercício profissional. Estudos de Psicologia, 1, 42-67.

Zanelli, J. C. (1994). Movimentos emergentes na prática dos psicólogos brasileiros nas organizações de trabalho: implicações para a formação. Em CFP, Psicólogo brasileiro: práticas emergentes e desafios para a formação (pp. 81156). São Paulo: Casa do Psicólogo.

Recebido em 08/03/2001

Revisado em 20/05/2001

Aceito em 23/05/2001 